\title{
Convolutional Neural Networks for High Throughput Screening of Catalyst Layer Inks for Polymer Electrolyte Fuel Cells
}

\author{
Mohammad J. Eslamibidgolia,*, Fabian P. Tipp ${ }^{\mathrm{b}}$, Jenia Jistev ${ }^{\mathrm{c}}$, Jasna \\ Jankovic $^{\mathrm{d}}$, Michael H. Eikerling ${ }^{\mathrm{a}, \mathrm{e}}$, Kourosh Malek ${ }^{\mathrm{a}, *}$ \\ ${ }^{a}$ Institute of Energy and Climate Research, Theory and Computation of Energy Materials \\ (IEK-13), Forschungszentrum Jülich, 52425 Jülich, Germany \\ ${ }^{b}$ Department of Chemistry, University of Cologne, Greinstr. 4-6, 50939 Cologne, Germany \\ ${ }^{c}$ Jülich Supercomputing Center, Forschungszentrum Jülich, 52425 Jülich, Germany \\ ${ }^{d}$ Department of Materials Science and Engineering, University of Connecticut, 97 \\ NorthEagleville Road, Unit 3136, Storrs, CT 06269-3136, USA \\ ${ }^{e}$ Chair of Theory and Computation of Energy Materials, Faculty of Georesources and \\ Materials Engineering, RWTH Aachen University, Aachen 52062, Germany
}

\begin{abstract}
The performance of polymer electrolyte fuel cells decisively depends on the structure and processes in membrane electrode assemblies and their components, in particular the catalyst layers. Essential structural building blocks of catalyst layers are formed during processing and application of catalyst inks. Accelerating the structural characterization at the ink stage is thus crucial to expedite further advances in catalyst layer design and fabrication. In this context, deep learning algorithms based on deep convolutional neural networks (ConvNets) can automate the processing of the complex and multi-scale structural features of ink imaging data. This article presents the first application of ConvNets for the high throughput screening of transmission electron microscopy images at the ink stage. Results indicate the importance of model pre-training and employment of data augmentation that works on multiple scales in the training of robust and accurate classification pipelines.
\end{abstract}

Keywords: High Throughput Screening, Catalyst Layers, Fuel Cells,

\footnotetext{
* Corresponding author

Email addresses: m.eslamibidgoli@fz-juelich.de (Mohammad J. Eslamibidgoli), k.malek@fz-juelich.de (Kourosh Malek)
} 
Microscopy Characterization, Deep Learning, Convolutional Neural Networks

\section{Introduction}

Despite decades of research, the rapid improvement of the effective electrocatalytic activity and durability of catalyst layers (CLs) remains a foremost challenge in efforts to drive forth the commercialization of polymer electrolyte

5 fuel cells (PEFCs). Accelerating the fabrication process and the evaluation of CLs are crucial in view of two major goals in PEFC development: drastically reduce the Pt loading and enable operation at high current and power density. [1, 2, 3, 4, 5, 6]

Conventional catalyst layers are fabricated from ink-based recipes that include a solid-state catalyst of Pt nanoparticles supported typically on a high-surface area carbon (Pt/C), an ionomer dispersion (e.g., Nafion or Aquivion), and a water-alcohol mixture as the solvent. [7, 8, 9, Ink-based methods allow for the rapid and reproducible manufacturing of membrane electrode assemblies. In the ink, $\mathrm{Pt} / \mathrm{C}$ particles assemble into porous agglomerates, with small intra15 agglomerate pores (primary pores, ; $10 \mathrm{~nm}$ ) and larger pores (secondary pores, $30-150 \mathrm{~nm}$ ) being formed in the inter-agglomerate space.[10, 11] Ionomer molecules assemble into a network of skin-type films that partially encapsulate agglomerates, with a thin proton-conducting water film formed between agglomerate surface and ionomer films. The resulting bi-modal pore size distribution as well as the heterogeneous wettability of pore surfaces determine the liquid water saturation in the CL as well as other porous fuel cell media, thereby exerting a major impact on PEFC performance. 12. Ink-based fabrication affords variations in Pt loading, ionomer content, carbon content and type of carbon, size distribution of $\mathrm{Pt}$ and $\mathrm{C}$ particles, wetting properties of pore walls, thickness of the applied CL, dielectric properties of the ink solvent, ink processing conditions, temperature, and stirring rate. In the past two decades, optimization in this multidimensional parameter space has been extensively pursued. 13, 14, 15, 16] Following ink fabrication, the ink is applied 
to the membrane or deposited onto a substrate by a wet-coating technique to prepare a catalyst coated membrane (CCM) or membrane electrode assembly (MEA). The properties of the starting ink, in combination with the CL deposition process, determine structure, properties and performance of resulting MEAs. [17, 18, 19, 20, 21] The correlations among fabrication parameters, ink ingredients, intrinsic materials properties, microstructure, and operating conditions are difficult to unravel. Large uncertainty and controversy thus remain regarding the key descriptors that must be monitored and controlled in the manufacturing process of MEAs to deliver cells with optimal performance.

Electron microscopy is the most widely employed characterization method in nanoscience. It provides images at (sub-) nanometer resolution, which con40 tain quantitative information about particle and pore size distributions, crystal structure, materials morphology, and composition. [22, 23] Transmission electron microscopy (TEM) has become a standard imaging technique to study the microstructure of MEA components, prior to cell assembly or post-testing. 24, 25, 26, 27, 28] High resolution imaging techniques such as high resolution TEM

45 (HR-TEM), high-angle annular dark field - scanning transmission electron microscopy (HAADF-STEM), 3D electron tomography or high-resolution atomic force microscopy (HR-AFM) have yielded 2D or 3D images of catalyst layer microstructures in both inks and deposited layers. 229, 30, 31, 32, 33, 34, 35. Many useful descriptors from TEM images of catalyst layer inks are difficult to distinguish upon visual examination. These features, however, can be automatically determined by machine learning algorithms. Moreover, recent advances in autonomous materials fabrications using lab-scale stationary or mobile robotic arms 36, 37, 38, 39, 40 demand a bidirectional and rapid data flow from catalyst fabrication to ink preparation, CCM fabrication, MEA assembly, and half or full cell design, including testing and in situ/ex situ characterization steps. The focus of this article is on the subsection of the discovery pipeline from the fuel cell ink to device. In this area, data workflows are lacking algorithms for extracting information from imaging data.41 An AI-driven algorithm for rapid image processing could assist materials discovery-on-a-chip by improving 
the data workflow. This approach, complemented by data acquisition units and physical modeling, as well as by high-throughput communication with AIdriven processes during the primary materials design stage, can be utilized for optimization purposes at all levels from ink, to CCM and further to complete cell or stack.

\section{Methods}

Our methodical pipeline for TEM image recognition of catalyst layer inks consists of three steps: (1) data sampling and data augmentation at multiple scales; (2) making use of a network model pre-trained on large generic natural images dataset, ImageNet, for transfer learning; and (3) training a classifier for ink

70 recognition. In the first step, the model performance is evaluated against the prepared fixed-scale datasets. In the second step, a comparison of model performance is made with a custom ConvNet model trained from scratch. Finally, in the last step we present a fast and accurate approach for ink classification by training a logistic regression model on extracted features obtained from transfer learning.

\subsection{Microscopy characterization}

For the purpose of our investigation, we used TEM imaging data provided by Automotive Fuel Cell Cooperation (AFCC) from two independent experimental studies of catalyst powder agglomeration with Nafion and Aquivion ionomers employed as hinders. Three ink powders were studied with Nafion ionomer, EA50 I33 ink, F50 I33 ink and V50 I33 ink (EA - graphitized, F - stabilized and V - Vulcan carbon support with Pt from TKK, and 33 wt\% Nafion ionomer), and three with Aquivion ionomer, EA50 I33 ink, F50E I33 ink and V50 I33 ink. The inks were sonicated for 30 minutes before deposition of a few drops onto a lacey carbon 300 mesh formvar coated TEM grid (TedPella). All samples were imaged using a FEI Tecnai G2 200 kV Transmission Electron Microscope. Further information about the raw images and initial processing are provided in the Electronic Supporting Information (ESI). 


\subsection{Dataset preparation}

90 The ink agglomerates encompass multi-scale structural features which are in fact highly relevant to the physical descriptors of CL performance and lifetime. As exemplified in Figure 1 (a) at different levels of magnification, these descriptors include the size, morphology and distribution of carbon particles as well as of catalyst nanoparticles, the size and distribution of ionomer aggregates, or the pore networks. As TEM images display structural features at different length scales and resolution, the main objective for a ConvNet model would be to effectively extract these features from the input data. Such features should make it possible for instance, to accurately identify the optimal vs. non-optimal samples or to reliably sort large databases in terms of the type of material. Therefore, during the training, the input dataset should be diverse enough to enable the emergence of such multi-scale structural features in the network.

To prepare a diverse input data space, we employed data augmentation techniques 42 ] via deploying region proposal algorithms - primarily designed for the object detection problems. 43] Two different region-specific approaches were employed: 1. A naïve and rather inefficient Sliding Window (SW) technique, and 2. the more efficient Selective Search (SS) algorithm. 43] By comparing the two approaches, we aim to demonstrate the effectiveness of using the latter. Given the high-resolution TEM ink data at large length scales (200-500 nm), these methods also generate a large number of examples for training and validation purposes required in the deep learning model development. Before applying the region-specific approaches, the images in each class were split into training and hold out sets; this ensures no leakage between the two sets (see ESI for details). As shown in Figure 1 (b), to sample the imaging data using the SW approach, varying window sizes of $100 \times 100,200 \times 200,300 \times 300$ and $500 \times 500$ pixels were slid over the raw ink images (see ESI). This was performed both on the training set and the hold out set. At each stop of the window, the corresponding fixed-scale image patch was extracted. This window was initiated at the top left of each image and moved 25-pixel steps to the right before moving back to the leftmost edge. After these steps, the window was moved down by 25 pixels 
(a)

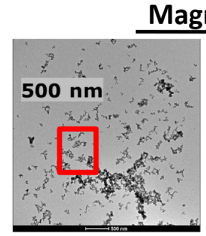

(c)
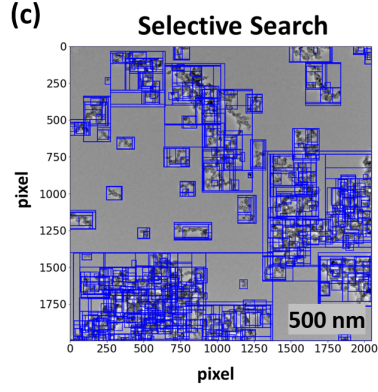
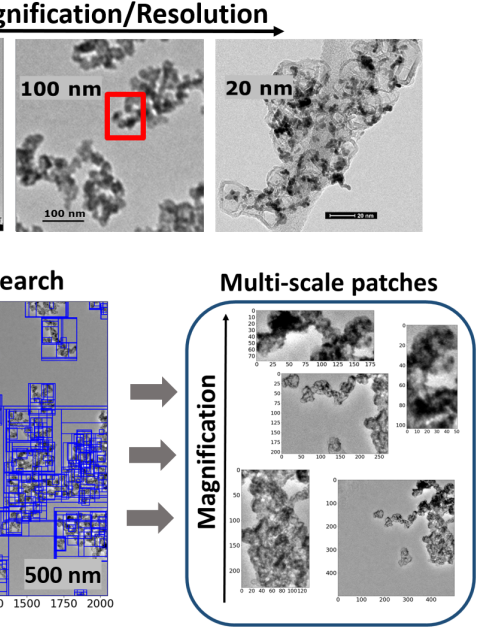

(b)

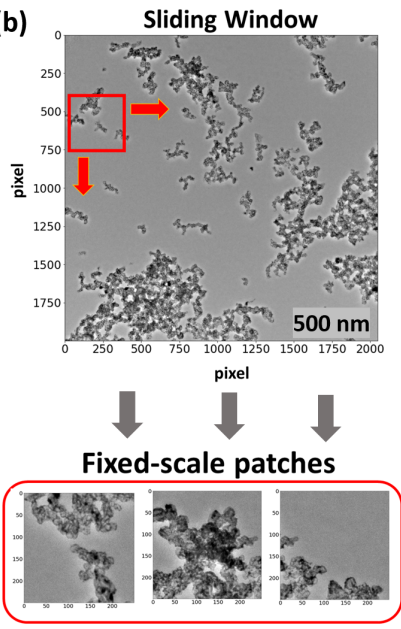

Figure 1: (a) An example of real-world images of catalyst ink agglomerates at different levels of magnification or resolution. (b) Representation of the Sliding Window technique to sample fixed-scale patches from high resolution TEM images of catalyst inks. (c) Representation of the Selective Search algorithm 43] to sample patches from high resolution TEM images of inks based on hierarchical grouping of similar regions such as color, size, texture and shape compatibility.

and this whole procedure was repeated. The main drawback of this technique is that it does not discriminate between parts of the images that contain the agglomerates from those that are mostly part of the background which leads to creating a noisy training set. Moreover, the choice for the aspect ratio of the sliding window is arbitrary; this may diminish the ability to extract useful features.

To address the first issue and filter out images with a high amount of background, only those images in which the mean grayscale value of the pixels is lower than 115-155 were saved, depending upon their respective classes (the possible values for the pixels range from 0 (black) to 255 (white)). This was performed on the basis that the agglomerates are significantly darker than the background, where the lower values of the pixels correspond to a darker tone. Therefore, a lower mean pixel value of an image generally corresponds to a larger 
proportion of the image being occupied by the agglomerates. After this initial step, the images were further inspected to remove images containing a large amount of background. As for the second issue, we used various window sizes as described above. Therefore, in our first approach to create the dataset, we combined several fixed- scale extracted patches using the SW technique, which is rather an inefficient way.

As shown in Figure 1 (c), as the second approach for data augmentation and extract patches from the high-resolution ink images, we employed the Selective Search algorithm. 43] Selective Search is a region proposal algorithm designed to localize possible object locations in the input image based on the hierarchical grouping of features and regions subject to the similarity of diverse factors such as the color, texture, lighting conditions, and composition - referred to diversification. Selective Search has outperformed several previous region proposal algorithms in terms of execution time, repeatability, proposal recall, detection and mean average precision. 44 On our dataset, it also resolved both issues with the SW approach, that is the undesired background patches and the lack of variability in the aspect ratio of the extracted images. This is because SS aims to select the parts of the image that contain agglomerates at different scales and with varying sizes.

A full implementation of the SS algorithm is available in Python, which includes three modes according to various diversification strategies explained in the original paper. 43. For our dataset preparation, the SS in the mode 'quality' was applied to each high-resolution ink image in the initially separated training and hold out sets. From this dataset, we discarded patches in which either length or width were under 100 pixels due to their relatively low resolution. Moreover, the ratio between the height and the width or between the width and the height was also calculated; all images where one of these two ratios are greater than 2 were discarded. From these resulting filtered images, about 2000 images for the training set and 2000 images for the hold out set were selected for each class (see Table 1 in the ESI). It was hereby ensured that the selected images were sourced in roughly equal proportions. 
(a)

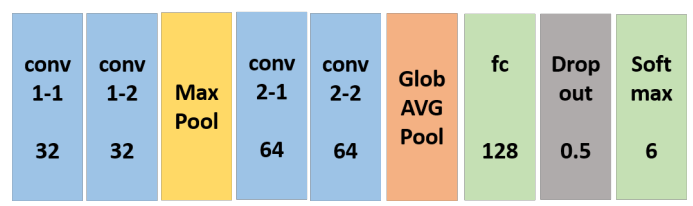

(b)

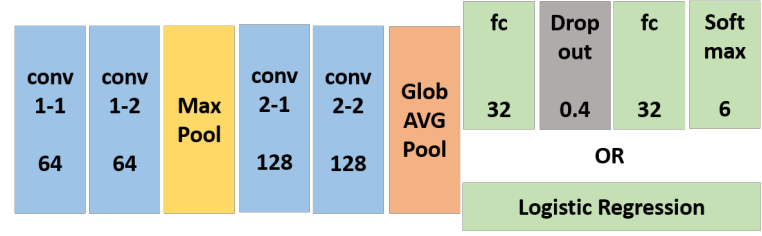

Figure 2: (a) Architectures of the custom network trained from scratch in this study, (b) that for the mini-VGG pretrained model on ImageNet dataset employed in this study for transfer learning for the catalyst ink image classification.

\subsection{ConvNets for image classification}


transformation that are essential for sufficient model expressiveness, while helping to avoid vanishing gradients during model optimization. Pooling constitutes a down-sampling process that operates over each activation map independently, creating a smaller activation map. The max pooling operation (pooling the maximum pixel value) is a common pooling operation alongside average pooling. Flattening or global average pooling are also applied to convert multiple feature maps to a single vector representation which is used as an input for a fully connected layer or classifier in the last step. [46] After a series of the convolutional layers, fully connected or global pooling layers are applied to generate a 1D feature vector. A classifier is then applied to generate the probability distribution over each class. In order to classify the image, the class corresponding to the largest probability value is taken. Once the class probability distribution is generated based on the current ConvNet weights, loss signaling mismatch between network output and true class assignments is computed. Backpropagation is then applied to compute the gradient of loss function with respect to each weight in every single kernel and the weights are modified to minimize the loss function.

Due to the high amount of training data, time, and computational resources required to train a ConvNet model from scratch transfer learning is usually adopted especially in cases where data is limited, where a pretrained model is used to extract reasonable generic features from the image and enable learning. A pretrained model can be for instance trained on a large generic natural image dataset to solve a classification problem. In our work, we employed the VGG16 model trained on the ImageNet dataset.47. For our dataset, we found that only the first four pretrained convolutional layers of VGG16 in conjunction with the corresponding max pooling layers are sufficient for accurate classification (miniVGG). The employed architectures are shown in Figure 2. 


\section{Results and Discussion}

210 The dataset was prepared for the six sample classes, namely, EA, F and V supports in Nafion and Aquivion. The overall steps to train and evaluate a ConvNet model after splitting and preprocessing the data include: 1. choosing the network architecture, 2. initializing the weights, 3. finding a learning rate and regularization strength, 4 . minimizing the loss function and monitoring the progress, and 5. evaluating the model on the hold out set.

To set a benchmark for our study, as a first approach, a relatively shallow custom ConvNet was constructed the architecture of which is shown in Figure 2 (a). Next, we evaluated various scenarios for the training and the hold out sets. Figure 3 shows the learning curves (validation accuracy vs. epoch number) of the different combinations. In the first case, when the network is trained on fixed-scale extracted patches of $100 \times 100$ using SW, it performs poorly on the hold out set generated using SS. In this case, the validation accuracy did not increase above $\approx 25 \%$ following 200 epochs indicating a significant overfitting for the model. Training our custom network on the dataset that generated 225 from SW including $100 \times 100,200 \times 200$ and $300 \times 300$ patches, increased the validation accuracy to $\approx 70 \%$ on the SS generated hold out set. This already suggests the importance of applying zoom-in and zoom-out data augmentation in the training phase. 48. Additionally, training on $100 \times 100,200 \times 200$ and $300 \times 300$ and $500 \times 500$ patches, further improved the performance to $\approx 75 \%$. If we train the network on the latter dataset from SW and test it on the SW generated hold out set, the accuracy further increases to $90 \%$. This is expected because as discussed SS selects patches at a different aspect ratio from that of SW. Therefore, it is not surprising to see an increased accuracy by $\approx 15 \%$ when both the training and hold out sets have similar aspect ratio. Our best validation accuracy, on the other hand, was obtained when we trained our custom network on the SS generated dataset. In this case, irrespective of how we prepared the hold out set (using SW or SS), a validation accuracy of $\approx 95 \%-97 \%$ was achieved. This suggests that, given a set of high-resolution ink images at large 


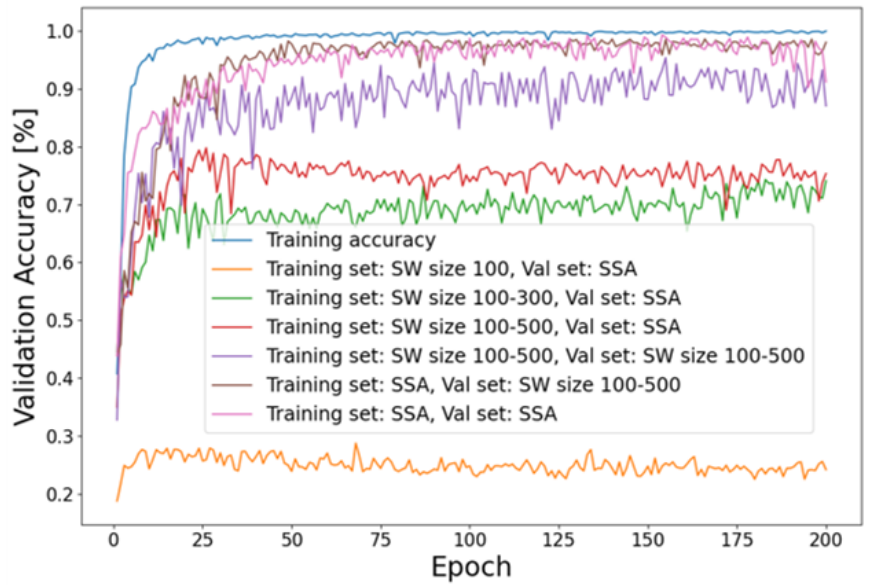

Figure 3: The learning curves for the validation accuracy of different scenarios of training and hold out sets considered in this study (SW: Sliding Window, SSA: Selective Search Algorithm).

length scale, SS can be effectively used to prepare training examples for training of the robust and accurate ink classification models.

Furthermore, a quicker approach was conducted, in which features were extracted from the first four convolutional layers of VGG16 (Figure 2 (b)), and then the feature vectors were used to train a logistic regression model for classification. Here, the first four convolutional layers of the pretrained VGG16 model were extracted along with two corresponding max pooling layers. At the end of the last layer, a global average pooling layer was added to obtain a feature vector. The training and validation images were passed through the network and the resulting feature vectors (128 entries per vector) were saved into numpy arrays. The features extracted from the training set were then used to fit a logistic regression model which is much faster than training neural networks. After this training, the labels of the hold out set were predicted and compared with the true labels for analysis. Figure 4 shows the normalized confusion matrix for the logistic regression classifier trained on the extracted features from the pretrained mini-VGG model. The $\mathrm{x}$-axis shows the predicted labels and the y-axis shows the true labels. The nearly diagonal matrix indicates the high accuracy of the model in classification of the six considered classes. 


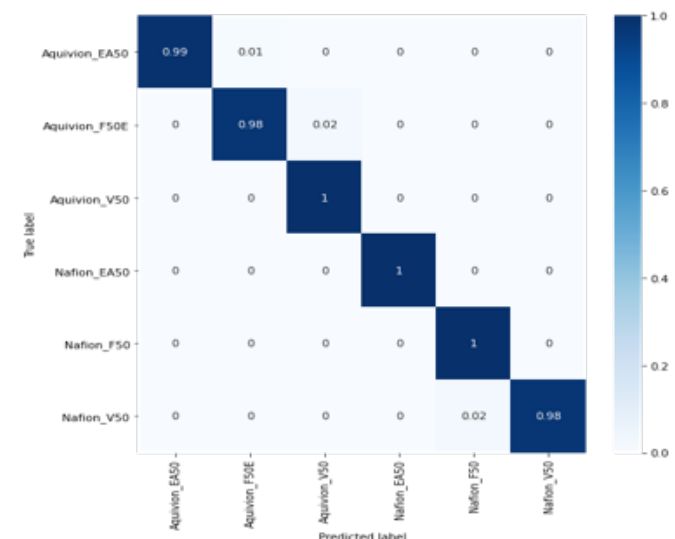

Figure 4: The confusion matrix obtained for classification of inks in 6 different sample categories using extracted features from the pretrained mini-VGG model followed by logistic regression as the classifier.

Table 1 compares the corresponding classification report in each class obtained from the pre-trained network with that from the custom network. Here, accuracy is the measure of true positive and true negative classifications over the sum of true positive, true negative, false positive and false negative. Precision is defined as the number of true positive (correctly labeled) classifications over the total true positive and false positive classifications. Recall is defined as the number of true positive classifications over the total number of true positives and false negatives. F1-score is the harmonic mean of precision and recall,

$$
F 1=2 \times \frac{\text { Precision } \times \text { Recall }}{\text { Precision }+ \text { Recall }}
$$

Comparing to the first approach of training the ConvNet model from scratch, our transfer learning approach not only was found to be swift, but also led to more accurate prediction, as summarized in Table 1. Thus, we conclude that preparing the training set with SS followed by employing pre-trained model for feature extraction and on top of that training the logistic regression model on for classification was found to be a fast and accurate process for this problem.

Gradient-weighted Class Activation Mapping (Grad-CAM) 49] was employed to visualize the important regions based on which our ConvNet model predicts the 

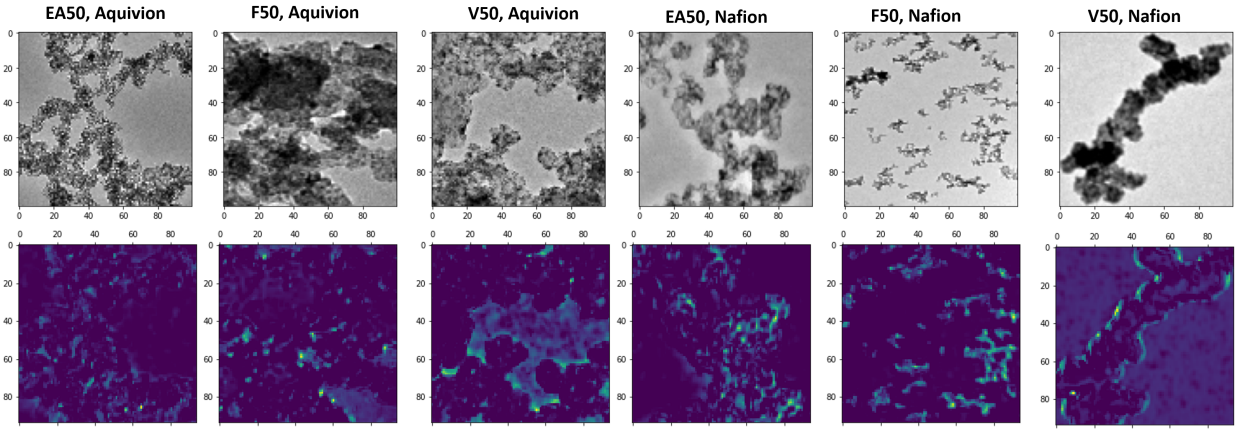

Figure 5: Grad-CAM visualization of the trained custom network 49] indicating the regions where our model interacts with ink images to classify them into six different sample categories.

Table 1: Inner-class evaluation of the logistic regression classifier on the hold out set; Features were extracted by pretrained mini-VGG backbone architecture. Values in parentheses correspond to the custom model trained from scratch.

\begin{tabular}{lllll}
\hline type & precision & recall & f1-score & num images \\
\hline Aquivion-EA50 & $1.00(0.96)$ & $0.98(0.96)$ & $0.99(0.96)$ & 1869 \\
Aquivion-F50E & $0.98(0.96)$ & $0.99(0.95)$ & $0.99(0.95)$ & 1890 \\
Aquivion-V50 & $0.99(0.95)$ & $0.99(0.97)$ & $0.99(0.96)$ & 1921 \\
Nafion-EA50 & $1.00(0.96)$ & $1.00(0.96)$ & $0.99(0.96)$ & 1863 \\
Nafion-F50 & $1.00(0.96)$ & $0.99(0.95)$ & $1.00(0.95)$ & 1882 \\
Nafion-V50 & $0.98(0.95)$ & $1.00(0.94)$ & $0.99(0.95)$ & 1607 \\
\hline
\end{tabular}

class of the ink agglomerates. It employs the class-specific gradient information from the last convolutional layer to produce class-discriminative activation maps. Highlighting these regions makes it easier to interpret how the ConvNet model interacts with ink images for classification. Figure 5 shows samples from each of the six different classes considered in our study along with their class-discriminative localization maps. First, it is important to note that the model does not predict the class based on unwanted features like the noise or the background color. In fact, it investigates the relevant regions of the image such as the edges of agglomerates where ionomer could be localized, the shape of the carbon agglomerates, the primary pores, and the Pt nanoparticles. As 
these features are highly relevant to the structure-function-property relations of catalyst layers in PEFCs, ConvNets can be considered highly promising for identification of optimal ink microstructures.

Overall, understanding how ConvNets learn from the of catalyst layer imaging data is a key to develop robust models for swift and accurate materials characterization. In the future, attempts should be made to generate a benchmark dataset of labelled TEM images of catalyst layer materials. Having a statistically representative dataset will provide essential input for ConvNets to further unravel the complex structure-property-function relations. Once trained over various fabrication formulations, operating criteria, and testing conditions, the presented approach based on ConvNet-based models can be used as a practical guideline for rapid screening and optimization of the catalyst ink compositions.

The presented work demonstrated an application of deep learning models based on convolutional neural networks for high throughput screening of transmission electron microscopy images of catalyst layer inks in the polymer electrolyte fuel cells. Results suggest that convolutional neural networks can significantly improve the efficacy of knowledge mining and analyses of ink imaging data by extracting relevant structural features that are difficult to distinguish upon visual examination. It was shown that robust and accurate models should be built upon data augmentation techniques to prepare a diverse input data of the ink images at multiple scales. These features comprise of crucial structural characteristics such as particle and pore size distributions and the ionomer cluster size distribution, which are related to the performance and the lifetime of catalyst layers. Indeed, visualizing the activation maps from the last convolutional layer of the trained models indicated that the class-discriminative features are extracted from the relevant physical descriptors. This would suggest that the convolutional neural networks are, for example, capable of rapid discrimination between optimal from non-optimal samples. 
While we specifically discussed the results in the context of the catalyst layer ink for polymer electrolyte fuel cells, the algorithms themselves are generic and can be trained for the similar fabrication processes, e.g. recognition of the slurry during fabrication of Li-ion electrodes. In the latter case, the algorithms will learn a different set of correlation factors and dependencies that govern the related structure-function relationships.

\section{Conflicts of interest}

The authors declare no competing financial interest.

\section{Acknowledgements}

The authors gratefully acknowledge the Gauss Centre for Supercomputing e.V. (www.gauss-centre.eu) for funding this project by providing computing time on the GCS Supercomputer JUWELS [50 at Jülich Supercomputing Centre (JSC) of Forschunszentrum Jülich. MJE, JJ, ME and KM acknowledge the financial support from Federal Ministry of Science and Education (BMBF) under German-Canadian Materials Acceleration Centre (GC-MAC) grant number 01DM21001A.

\section{References}

[1] W. Vielstich, A. Lamm, H. Gasteiger, Handbook of fuel cells. fundamentals, technology, applications.

[2] M. Eikerling, A. A. Kornyshev, A. R. Kucernak, Water in polymer electrolyte fuel cells: Friend or foe?, Physics today 59 (10) (2006) 38.

[3] M. Eikerling, A. A. Kornyshev, A. A. Kulikovsky, Physical modeling of fuel cells and their components, Encyclopedia of Electrochemistry: Online.

[4] M. Uchida, Y. Aoyama, N. Eda, A. Ohta, Investigation of the microstructure in the catalyst layer and effects of both perfluorosulfonate ionomer and 
ptfe-loaded carbon on the catalyst layer of polymer electrolyte fuel cells, Journal of the Electrochemical Society 142 (12) (1995) 4143.

[5] M. Uchida, Y. Fukuoka, Y. Sugawara, N. Eda, A. Ohta, Effects of mi-

[10] E. Sadeghi, A. Putz, M. Eikerling, Hierarchical model of reaction rate distributions and effectiveness factors in catalyst layers of polymer electrolyte fuel cells, Journal of the Electrochemical Society 160 (10) (2013) F1159.

[11] A. Malekian, S. Salari, J. Stumper, N. Djilali, M. Bahrami, Effect of compression on pore size distribution and porosity of pem fuel cell catalyst lay-

[12] T. Muzaffar, T. Kadyk, M. Eikerling, Tipping water balance and the pt loading effect in polymer electrolyte fuel cells: a model-based analysis, Sustainable Energy \& Fuels 2 (6) (2018) 1189-1196. 
[13] M. H. Eikerling, K. Malek, Q. Wang, Catalyst layer modeling: Structure,

[14] S. Holdcroft, Fuel cell catalyst layers: a polymer science perspective, Chemistry of materials 26 (1) (2014) 381-393.

[15] M. Chen, C. Zhao, F. Sun, J. Fan, H. Li, H. Wang, Research progress properties and performance, in: PEM fuel cell electrocatalysts and catalyst layers, Springer, 2008, pp. 381-446. of catalyst layer and interlayer interface structures in membrane electrode assembly (mea) for proton exchange membrane fuel cell (pemfc) system, eTransportation (2020) 100075.

[16] A. Malek, E. Sadeghi, J. Jankovic, M. Eikerling, K. Malek, Aquivion ionomer in mixed alcohol-water solution: insights from multiscale molecular modeling, The Journal of Physical Chemistry C 124 (6) (2020) 34293438 .

[17] B. Garlyyev, K. Kratzl, M. Rück, J. Michalička, J. Fichtner, J. M. Macak, T. Kratky, S. Günther, M. Cokoja, A. S. Bandarenka, et al., Optimizing the size of platinum nanoparticles for enhanced mass activity in the electrochemical oxygen reduction reaction, Angewandte Chemie International Edition 58 (28) (2019) 9596-9600.

[18] H. Ishikawa, Y. Sugawara, G. Inoue, M. Kawase, Effects of pt and ionomer ratios on the structure of catalyst layer: A theoretical model for polymer electrolyte fuel cells, Journal of Power Sources 374 (2018) 196-204.

[19] S. A. Berlinger, B. D. McCloskey, A. Z. Weber, Inherent acidity of perfluorosulfonic acid ionomer dispersions and implications for ink aggregation, The Journal of Physical Chemistry B 122 (31) (2018) 7790-7796.

[20] J. Hou, M. Yang, C. Ke, G. Wei, C. Priest, Z. Qiao, G. Wu, J. Zhang, Platinum-group-metal catalysts for proton exchange membrane fuel cells: From catalyst design to electrode structure optimization, EnergyChem 2 (1) (2020) 100023. 
[21] T. Soboleva, K. Malek, Z. Xie, T. Navessin, S. Holdcroft, Pemfc catalyst layers: the role of micropores and mesopores on water sorption and fuel cell activity, ACS applied materials \& interfaces 3 (6) (2011) 1827-1837.

[22] D. Su, Advanced electron microscopy characterization of nanomaterials for catalysis, Green Energy \& Environment 2 (2) (2017) 70-83.

[23] D. H. Anjum, Characterization of nanomaterials with transmission electron microscopy, in: IOP Conference Series: Materials Science and Engineering, Vol. 146, IOP Publishing, 2016, p. 012001.

[24] J. Jankovic, Latest advances in $2 \mathrm{~d}$ and $3 \mathrm{~d}$ microstructural characterization of fuel cell components, in: ECS Meeting Abstracts, no. 38, IOP Publishing, 2019, p. 1767.

[25] S. Pedram, N. Macauley, S. Zhong, H. Xu, J. Jankovic, Microscopy studies of the catalyst inks for pem fuel cells, ECS Transactions 98 (9) (2020) 197.

[26] J. Jankovic, D. Susac, T. Soboleva, J. Stumper, Electron tomography based 3d reconstruction of fuel cell catalysts, ECS Transactions 50 (2) (2013) 353.

[27] J. Chen, A. Goshtasbi, A. P. Soleymani, M. Ricketts, J. Waldecker, C. Xu, J. Yang, T. Ersal, J. Jankovic, Effects of cycle duration and test hardware in relative humidity cycling of a polymer electrolyte membrane, Journal of Power Sources 476 (2020) 228576.

[28] L. G. Melo, A. P. Hitchcock, J. Jankovic, J. Stumper, D. Susac, V. Berejnov, Quantitative mapping of ionomer in catalyst layers by electron and x-ray spectromicroscopy, ECS Transactions 80 (8) (2017) 275.

[29] M. Sabharwal, L. Pant, A. Putz, D. Susac, J. Jankovic, M. Secanell, Analysis of catalyst layer microstructures: From imaging to performance, Fuel Cells 16 (6) (2016) 734-753.

[30] R. L. Borup, A. Kusoglu, K. C. Neyerlin, R. Mukundan, R. K. Ahluwalia, D. A. Cullen, K. L. More, A. Z. Weber, D. J. Myers, Recent developments 
in catalyst-related pem fuel cell durability, Current Opinion in Electrochemistry 21 (2020) 192-200.

[31] Y.-C. Park, H. Tokiwa, K. Kakinuma, M. Watanabe, M. Uchida, Effects of carbon supports on pt distribution, ionomer coverage and cathode performance for polymer electrolyte fuel cells, Journal of Power Sources 315 (2016) 179-191.

[32] R. Hiesgen, T. Morawietz, M. Handl, M. Corasaniti, K. A. Friedrich, Atomic force microscopy on cross sections of fuel cell membranes, electrodes, and membrane electrode assemblies, Electrochimica Acta 162 (2015) 86-99.

[33] S. Helmly, M. Eslamibidgoli, K. A. Friedrich, M. Eikerling, Local impact of pt nanodeposits on ionomer decomposition in polymer electrolyte membranes, Electrocatalysis 8 (6) (2017) 501-508.

[34] D. Rossouw, L. Chinchilla, N. Kremliakova, G. A. Botton, The 3d nanoscale evolution of platinum-niobium oxide fuel cell catalysts via identical location electron tomography, Particle \& Particle Systems Characterization 34 (7) (2017) 1700051.

[35] H. Uchida, J. M. Song, S. Suzuki, E. Nakazawa, N. Baba, M. Watanabe, Electron tomography of nafion ionomer coated on pt/carbon black in high utilization electrode for pefcs, The Journal of Physical Chemistry B 110 (27) (2006) 13319-13321.

[36] A. Malek, M. J. Eslamibidgoli, M. Mokhtari, Q. Wang, M. H. Eikerling, K. Malek, Virtual materials intelligence for design and discovery of advanced electrocatalysts, ChemPhysChem 20 (22) (2019) 2946-2955.

[37] B. Burger, P. M. Maffettone, V. V. Gusev, C. M. Aitchison, Y. Bai, X. Wang, X. Li, B. M. Alston, B. Li, R. Clowes, et al., A mobile robotic 445 chemist, Nature 583 (7815) (2020) 237-241. 
[38] R. Shimizu, S. Kobayashi, Y. Watanabe, Y. Ando, T. Hitosugi, Autonomous materials synthesis by machine learning and robotics, APL Materials 8 (11) (2020) 111110.

[39] B. P. MacLeod, F. G. Parlane, T. D. Morrissey, F. Häse, L. M. Roch, K. E. Dettelbach, R. Moreira, L. P. Yunker, M. B. Rooney, J. R. Deeth, et al., Self-driving laboratory for accelerated discovery of thin-film materials, Science Advances 6 (20) (2020) eaaz8867.

[40] C. W. Coley, N. S. Eyke, K. F. Jensen, Autonomous discovery in the chemical sciences part i: Progress, Angewandte Chemie International Edition 59 (51) (2020) 22858-22893.

[41] M. Ge, F. Su, Z. Zhao, D. Su, Deep learning analysis on microscopic imaging in materials science, Materials Today Nano 11 (2020) 100087.

[42] E. D. Cubuk, B. Zoph, D. Mane, V. Vasudevan, Q. V. Le, Autoaugment: Learning augmentation policies from data, arXiv preprint arXiv:1805.09501.

[43] J. R. Uijlings, K. E. Van De Sande, T. Gevers, A. W. Smeulders, Selective search for object recognition, International journal of computer vision 104 (2) (2013) 154-171.

[44] S. B. Hosang J, Benenson R, How good are detection proposals, really?, arXiv preprint arXiv:1406.6962.

[45] A. Krizhevsky, I. Sutskever, G. E. Hinton, Imagenet classification with deep convolutional neural networks, Advances in neural information processing systems 25 (2012) 1097-1105.

[46] Y. Guo, Y. Liu, A. Oerlemans, S. Lao, S. Wu, M. S. Lew, Deep learning 470 for visual understanding: A review, Neurocomputing 187 (2016) 27-48.

[47] K. Simonyan, A. Zisserman, Very deep convolutional networks for largescale image recognition, arXiv preprint arXiv:1409.1556. 
[48] Y. Chen, Y. Li, T. Kong, L. Qi, R. Chu, L. Li, J. Jia, Scale-aware automatic augmentation for object detection, in: Proceedings of the IEEE/CVF Conference on Computer Vision and Pattern Recognition, 2021, pp. 9563-9572.

[49] R. R. Selvaraju, M. Cogswell, A. Das, R. Vedantam, D. Parikh, D. Batra, Grad-cam: Visual explanations from deep networks via gradient-based localization, in: Proceedings of the IEEE international conference on computer vision, 2017, pp. 618-626.

[50] D. Krause, Juwels: Modular tier-0/1 supercomputer at the jülich supercomputing centre, Journal of large-scale research facilities JLSRF 5 (2019) 135. 